\title{
Hepatopathy of unknown etiology - is liver biopsy a good tool in differential diagnosis?
}

Joanna Jabłońska ${ }^{1}$, Joanna Cielecka-Kuszyk ${ }^{2}$, Tomasz Mikuła ${ }^{1}$, Joanna Kozłowska ${ }^{1}$, Alicja Wiercińska-Drapało ${ }^{1}$

\author{
${ }^{1}$ Department of Infectious and Tropical Diseases and Hepatology, Medical University \\ of Warsaw, Warsaw, Poland \\ ${ }^{2}$ Department of Virology, National Institute of Public Health - National Institute \\ of Hygiene, Warsaw, Poland
}

Submitted: 25 February 2017

Accepted: 14 May 2017

Arch Med Sci 2019; 15 (6): 1462-1467

DOI: https://doi.org/10.5114/aoms.2019.82637

Copyright $\odot 2019$ Termedia \& Banach

\section{Abstract}

Introduction: Liver biopsy is a well-known method for the diagnosis and evaluation of chronic diffuse liver diseases, especially among patients with "hepatopathy of unknown origin".

Material and methods: In the years 2014-2015 we performed 259 liver biopsies in 28 patients (22 females, 6 males, aged 18-65 years, mean: 45 years) with an initial diagnosis of "hepatopathy of unknown origin". The liver biopsies of these 28 patients were revised by two independent pathologists. Results: Histopathological features of autoimmune conditions were found in 11 cases, steatohepatitis with/without Mallory bodies in 7, simple steatosis without inflammation in 2 cases. In the other 8 cases the histopathological features were non-specific but pointed to vanishing bile duct syndrome, hemochromatosis, acute inflammation or fibrosis without inflammation. Surprisingly, only mild fibrosis without inflammatory infiltrates was present in one patient with a high titer of antinuclear antibodies (ANA > $1: 3200$ ). Mild cholestasis with bilirubinostasis was found in 4 cases. One patient had prominent lobular iron deposits and is now under observation for hemochromatosis. Vanishing bile duct syndrome as ductopenia without any signs of inflammation was found in one patient with suspicion of primary biliary cirrhosis. In one liver biopsy specimen we found normal liver architecture without inflammation or steatosis in a patient with elevated ALT and GGT, negative for viral antibodies and autoantibodies.

Conclusions: Liver biopsy - despite the increasing access to new, non-invasive methods - remains a useful method in the differential diagnosis of liver diseases.

Key words: liver biopsy, hepatopathy of unknown origin, ultrasound elastography.

\section{Introduction}

Liver biopsy has been considered as a gold standard for the diagnosis and evaluation of chronic diffuse liver diseases for many years, despite the fact that this is an invasive test and complications can occur. Several potential limitations of the liver biopsy have been described, such as the fact, that various areas of the liver can be affected to different degrees, sampling errors, discordance in the interpretation of

\author{
Corresponding author: \\ Dr. Tomasz Mikuła \\ Department \\ of Infectious and \\ Tropical Diseases \\ and Hepatology \\ Medical University \\ of Warsaw \\ 37 Wolska St \\ 01-201 Warsaw, Poland \\ Phone: +48 602416791 \\ E-mail: tomasz.mikula6@ \\ wp.pl
}


the histological findings or lack of acceptability of the biopsy by the patient [1]. Usually the first liver biopsy is recommended to establish the diagnosis of chronic liver diseases and consecutive biopsies are sometimes necessary to evaluate hepatic changes in the course of the disease, as aminotransferase activity is not always a reliable marker for inflammation and fibrosis [2] and the FibroTest and transient elastography assess only fibrosis $[3,4]$.

We examined retrospectively selected material to assess the usefulness of liver biopsy in the differential diagnosis in patients with liver disease of unknown origin.

\section{Material and methods}

In the years 2014-2015 we performed 259 liver biopsies in the Department of Infectious and Tropical Diseases and Hepatology of Medical University of Warsaw. In 28 patients (22 females, 6 males, aged 18-65 years, mean: 45 years) the initial diagnosis was "hepatopathy of unknown origin". The liver biopsies of these 28 patients were reviewed by two independent pathologists.

The liver biopsy specimens were fixed in $10 \%$ buffered formalin, processed routinely into paraffin blocks and stained with H\&E, Chromotrope $2 R$ and Gomori silver stain. In all biopsies the material was representative, displaying at least 12 complete portal spaces with adjacent parenchyma. Histological grade and stage as inflammatory activity and fibrosis or steatosis were assessed according to the Batts-Ludwig and Brunt classification.

The histological changes in primary sclerosing cholangitis (PSC) were divided into four stages: I. Portal stage: Mixed inflammatory cell infiltrates composed of lymphocytes, plasma cells and neutrophils, more intense around the bile ducts, presence of lymphoid follicles and very occasionally granulomas, degenerative epithelial changes of small bile ducts, increase portal fibrosis; II. Periportal stage: Disruption of the limiting plate, biliary interface activity, focal ductular proliferation, sometimes dense portal tract inflammation, periductal fibrosis, cholangitis and focal ductopenia; III. Septal stage: marked portal fibrosis, portal portal septa; IV. Cirrhosis, absence of bile ducts and rounded scars (Ludwig).

The histological changes in autoimmune hepatitis (AlH) were scored using the four-degree scale for the grade (inflammatory activity) and stage (fibrosis) of the disease. The grade was assessed as: 0 - portal inflammation only, without piecemeal necrosis, without lobular inflammation and necrosis; 1 - minimal portal inflammation, minimal, patchy piecemeal necrosis, occasional spotty necrosis; 2 - mild portal inflammation, mild piecemeal necrosis involving some or all portal tracts, little hepatocellular damage; 3 - moderate portal inflammation, moderate piecemeal necrosis involving all portal tracts, moderate lobular inflammation with noticeable hepatocellular change; 4 - severe portal inflammation, severe piecemeal necrosis, may have bridging necrosis, severe lobular inflammation with prominent diffuse hepatocellular damage. The stage was assessed as: 0 - no fibrosis, normal connective tissue; 1 - portal fibrosis, fibrous portal expansion; 2 - periportal fibrosis, periportal or rare portal-portal septa; 3 - septal fibrosis, fibrous septa with architectural distortion; 4 - cirrhosis.

The histological changes, including the presence of epithelioid cell granuloma, in PBC were described as stage I: florid duct lesions, portal hepatitis; stage II: ductular proliferation and periportal fibrosis, stage III: bridging necrosis, septal fibrosis; stage IV: cirrhosis. The histological changes in autoimmune chronic hepatitis (AICh, toxic hepatitis) were scored using the four-degree scale for the grade (inflammatory activity) and stage (fibrosis) of the disease.

According to Brunt, grading activity and staging fibrosis in non-alcoholic steatohepatitis (NASH) and alcoholic steatohepatitis (ASH) were assessed as mild with steatosis $<30 \%$ and minimal hepatocyte ballooning, moderate with steatosis of 30$65 \%$ and ballooning present, severe with steatosis $>66 \%$ and marked hepatocyte ballooning.

\section{Results}

The laboratory and histological features are presented in Tables I and II.

Histopathological features of autoimmune conditions were found as probable in 11 (38\%) cases, steatohepatitis with/without Mallory bodies in $7(25 \%)$ cases, simple steatosis without inflammation in 2 (7\%) cases. In the remaining 8 (30\%) cases the histopathological features were non-specific but pointed to vanishing bile duct syndrome, hemochromatosis, acute inflammation or fibrosis without inflammation. Surprisingly, only mild fibrosis without inflammatory infiltrates was present in one patient with a high titer of antinuclear antibodies (ANA > $1: 3200$ ). Mild cholestasis with bilirubinostasis was found in 4 cases. One patient had prominent lobular iron deposits and is now under observation for hemochromatosis. Vanishing bile duct syndrome as ductopenia without any signs of inflammation was found in one patient with suspicion of primary biliary cirrhosis (PBC). In one liver biopsy specimen we have found normal liver architecture without inflammation or steatosis in a patient with elevated alanine aminotransferase (ALT) and $\gamma$-glutamyltransferase (GGT), negative for viral antibodies and autoantibodies (Figures 1-3). 
Table I. Baseline characteristics of analyzed group - females $(n=22)$

\begin{tabular}{|c|c|c|c|c|c|c|c|c|}
\hline $\begin{array}{l}\text { Patients } \\
\text { no. }\end{array}$ & $\begin{array}{c}\text { Age } \\
\text { [years] }\end{array}$ & $\begin{array}{l}\text { AST (norm: } \\
10-59 \mathrm{U} / \mathrm{l})\end{array}$ & $\begin{array}{l}\text { ALT (norm: } \\
10-70 \mathrm{U} / \mathrm{l})\end{array}$ & $\begin{array}{l}\text { GGT (norm: } \\
15-73 \mathrm{U} / \mathrm{l})\end{array}$ & $\begin{array}{l}\text { ALP (norm: } \\
38-126 \mathrm{U} / \mathrm{l})\end{array}$ & $\begin{array}{l}\text { HBV } \\
\text { status }\end{array}$ & $\begin{array}{c}\mathrm{HCV} \\
\text { status }\end{array}$ & $\begin{array}{l}\text { Histopathological } \\
\text { results }\end{array}$ \\
\hline 1 & 38 & 94 & 127 & 112 & 62 & Neg & Neg & $\begin{array}{l}\text { Steatohepatitis, } \\
\text { Mallory bodies }\end{array}$ \\
\hline 2 & 43 & 67 & 92 & 628 & 200 & Neg & Neg & $\mathrm{AlH} / \mathrm{PSC}$ \\
\hline 3 & 45 & 54 & 63 & 303 & 179 & Neg & Neg & NASH \\
\hline 4 & 52 & 32 & 33 & 74 & 100 & Neg & $\mathrm{Neg}$ & $\begin{array}{l}\text { Mild fibrosis, } \\
\text { inconclusive }\end{array}$ \\
\hline 5 & 47 & 196 & 535 & 69 & 131 & Neg & Neg & NASH \\
\hline 6 & 55 & 1122 & 1368 & 183 & 167 & Neg & Neg & $\mathrm{AlH}$ \\
\hline 7 & 65 & 1265 & 888 & 149 & 263 & Neg & $\mathrm{Neg}$ & $\begin{array}{l}\text { Chronic hepatitis- } \\
\text { hemochromatosis }\end{array}$ \\
\hline 8 & 18 & 38 & 54 & 66 & 111 & Neg & Neg & Toxic hepatitis \\
\hline 9 & 42 & 600 & 1100 & 71 & 139 & Neg & Neg & $\mathrm{AlH}$ \\
\hline 10 & 29 & 243 & 410 & 106 & 168 & Neg & Neg & AlH \\
\hline 11 & 55 & 71 & 73 & 191 & 105 & Neg & $\mathrm{Neg}$ & $\begin{array}{c}\text { Ductopenia/ } \\
\text { vanishing bile duct } \\
\text { syndrome }\end{array}$ \\
\hline 12 & 32 & 41 & 60 & 334 & 425 & Neg & Neg & $\begin{array}{l}\mathrm{PBC} / \mathrm{AlCh} / \mathrm{lgG} 4 \\
\text { cholangitis }\end{array}$ \\
\hline 13 & 59 & 46 & 57 & 153 & 77 & Neg & SVR & PSC \\
\hline 14 & 60 & 156 & 548 & 376 & 240 & Neg & $\mathrm{Neg}$ & $\begin{array}{l}\text { Chronic hepatitis, } \\
\text { probable toxic }\end{array}$ \\
\hline 15 & 31 & 123 & 110 & 131 & 103 & Neg & Neg & AlH \\
\hline 16 & 55 & 93 & 119 & 26 & 51 & Neg & Neg & NASH \\
\hline 17 & 31 & 365 & 739 & 58 & 97 & Neg & $\mathrm{Neg}$ & $\begin{array}{l}\text { Acute hepatitis, } \\
\text { without fibrosis }\end{array}$ \\
\hline 18 & 34 & 60 & 86 & 200 & 127 & Neg & Neg & $\mathrm{AlH} / \mathrm{PSC}$ \\
\hline 19 & 26 & 136 & 198 & 548 & 449 & Neg & Neg & PSC \\
\hline 20 & 45 & 46 & 62 & 142 & 161 & Neg & Neg & NASH \\
\hline 21 & 57 & 48 & 63 & 192 & 193 & $\begin{array}{l}\text { HBcAb } \\
\text { pos }\end{array}$ & $\mathrm{Neg}$ & $\mathrm{PBC} / \mathrm{AICh}$ \\
\hline 22 & 59 & 400 & 600 & 200 & 265 & Neg & $\mathrm{Neg}$ & $\mathrm{AlH}$ \\
\hline
\end{tabular}

AST - aspartate transaminase, ALT - alanine transaminase, GGT- $\gamma$-glutamyl transpeptidase, ALP-alkaline phosphatase, HBV-hepatitis $B$ virus, $H C V$ - hepatitis C virus, AlH - autoimmune hepatitis, AlCh - autoimmune cholangitis, NASH - nonalcoholic steatohepatitis, neg negative, pos - positive, $P B C$ - primary biliary cirrhosis, $P S C$ - primary sclerosing cholangitis, SVR - sustain viral response - post anti-HCV treatment, $H B C A b-$ hepatitis $B$ virus core antibody, $\lg G 4-\lg G 4$-related cholangitis.

\section{Discussion}

The evaluation of chronic liver diseases requires several investigations including biological and clinical evaluation, as well as pathological examination of the liver, for making the diagnosis and establishing the grading and staging of the disease. It is still considered that the liver biopsy plays a key role for the diagnosis and follow-up of chronic diffuse hepatopathies and enables one to exclude another hepatopathy or an associated disease [5-7]. The analysis of a large cohort of 2413 patients showed that the liver biopsy changed the diagnosis in $31.2 \%$ of cases and was a safe procedure $[8,9]$. The main indicators for the liver biopsy include diagnostic and prognostic purposes, evaluating disease severity and monitoring response to treatment, together with the diagnostic performance of non-invasive methods [10-12]. It is an irreplaceable tool in diagnosis and follow-up of several chronic liver diseases [13]. In our retrospective study, the liver biopsy was performed in 259 patients with chronic liver disease. From this group, 29 (11\%) were considered as hepatopathy of unknown etiology. Liver biopsy changed the diagnosis and helped in performing clinical man- 
Table II. Baseline characteristics of analyzed group - males $(n=6)$

\begin{tabular}{|lcccccccc|}
\hline $\begin{array}{l}\text { Patients } \\
\text { no. }\end{array}$ & $\begin{array}{c}\text { Age } \\
\text { [years] }\end{array}$ & $\begin{array}{c}\text { AST (norm: } \\
10-59 \text { U/l) }\end{array}$ & $\begin{array}{c}\text { ALT (norm: } \\
10-70 ~ U / l)\end{array}$ & $\begin{array}{c}\text { GGT (norm: } \\
15-73 \text { U/l) }\end{array}$ & $\begin{array}{c}\text { ALP (norm: } \\
\mathbf{3 8 - 1 2 6} \text { U/l) }\end{array}$ & $\begin{array}{c}\text { HBV } \\
\text { status }\end{array}$ & $\begin{array}{c}\text { HCV } \\
\text { status }\end{array}$ & $\begin{array}{c}\text { Histopathological } \\
\text { results }\end{array}$ \\
\hline 1 & 34 & 32 & 117 & 45 & 59 & Neg & Neg & $\begin{array}{c}80 \% \text { hepatocyte } \\
\text { steatosis }\end{array}$ \\
\hline 2 & 35 & 320 & 340 & 30 & 200 & Neg & Neg & $\begin{array}{c}\text { Cholestasis, } \\
\text { hepatitis - probable } \\
\text { toxic }\end{array}$ \\
\hline 3 & 33 & 60 & 115 & 66 & 120 & Neg & Neg & NASH \\
\hline 4 & 26 & 65 & 124 & 146 & 44 & Neg & Neg & NASH \\
\hline 5 & 48 & 30 & 59 & 145 & 104 & Neg & Neg & $\begin{array}{c}40 \% \text { hepatocyte } \\
\text { steatosis }\end{array}$ \\
\hline 6 & 39 & 50 & 199 & 260 & 134 & Neg & Neg & Norm \\
\hline
\end{tabular}

AST - aspartate transaminase, ALT - alanine transaminase, GGT- $\gamma$-glutamyl transpeptidase, ALP - alkaline phosphatase, HBV - hepatitis $B$ virus, $H C V$ - hepatitis C virus, NASH - nonalcoholic steatohepatitis, neg-negative.
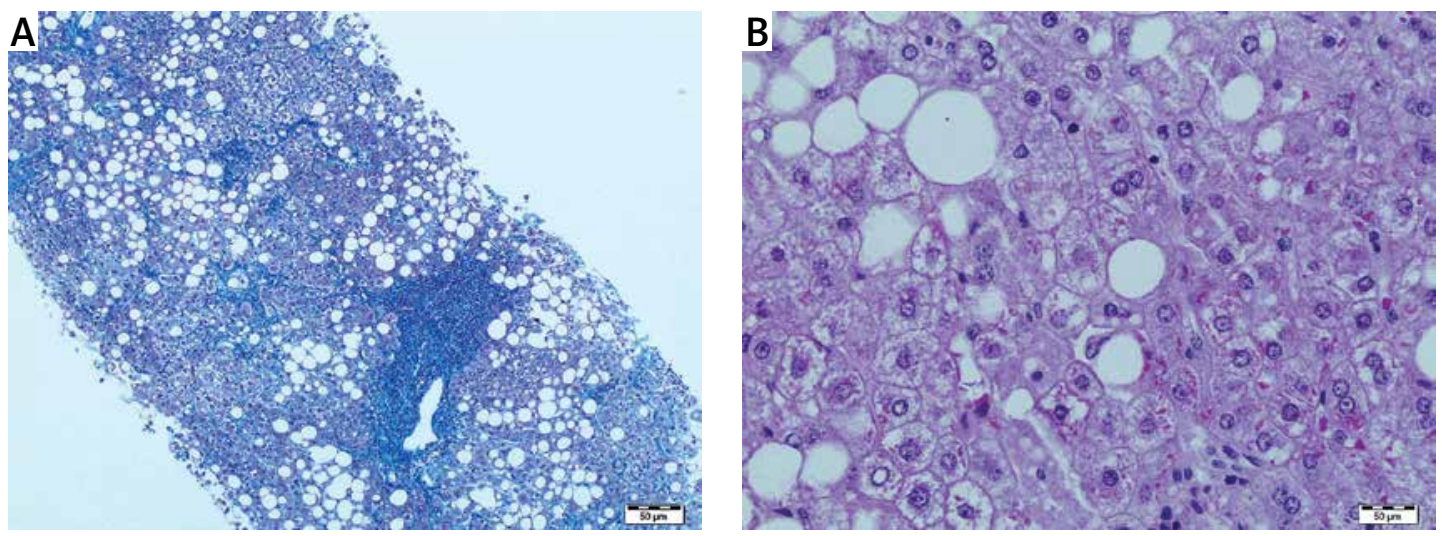

Figure 1. Patient 1 (female) (A and B): steatohepatitis, macrovesicular steatosis, mild fibrosis and portal inflammation
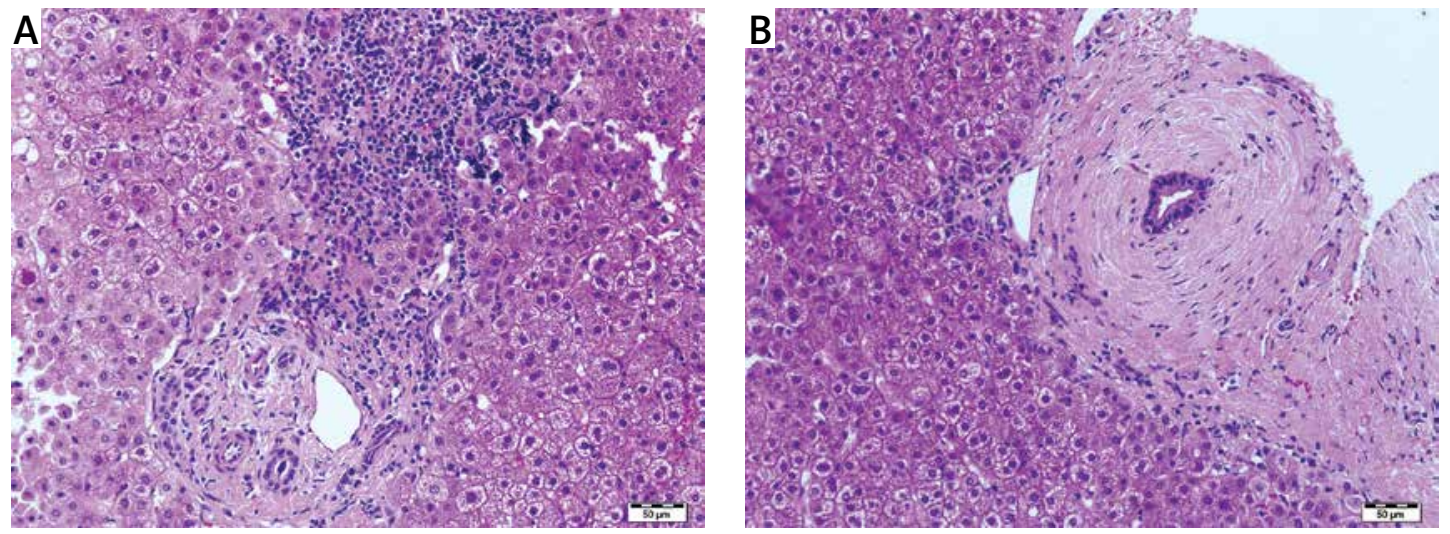

Figure 2. Patient 2 (female) (A and B): autoimmune hepatitis/primary sclerosing cholangitis

agement in 20 (70\%) patients. Liver biopsy was an effective method for approaching the diagnosis of autoimmune conditions, non/alcoholic fatty liver disease and cholangiopathy features. The abundant inflammatory infiltrates of plasma cells, piecemeal necrosis, lymphocyte rosetting and lobular plasma cell infiltrates correlated with the diagnosis of autoimmune hepatitis (AlH). The presence of non-suppurative destructive cholangitis with granuloma formations shown in the primary biliary cirrhosis and the concentric periductal fibrosis was a marker of primary sclerosing cholangitis, which correlated with other clinical findings. Liver biopsy was a useful tool in the diagnosis of overlap syndromes such as AIH/PSC and AIH/PBC. Our study confirms the recommendations for liver biopsy to establish the diagnosis in autoimmune patients, and enables the diagnosis of overlap 

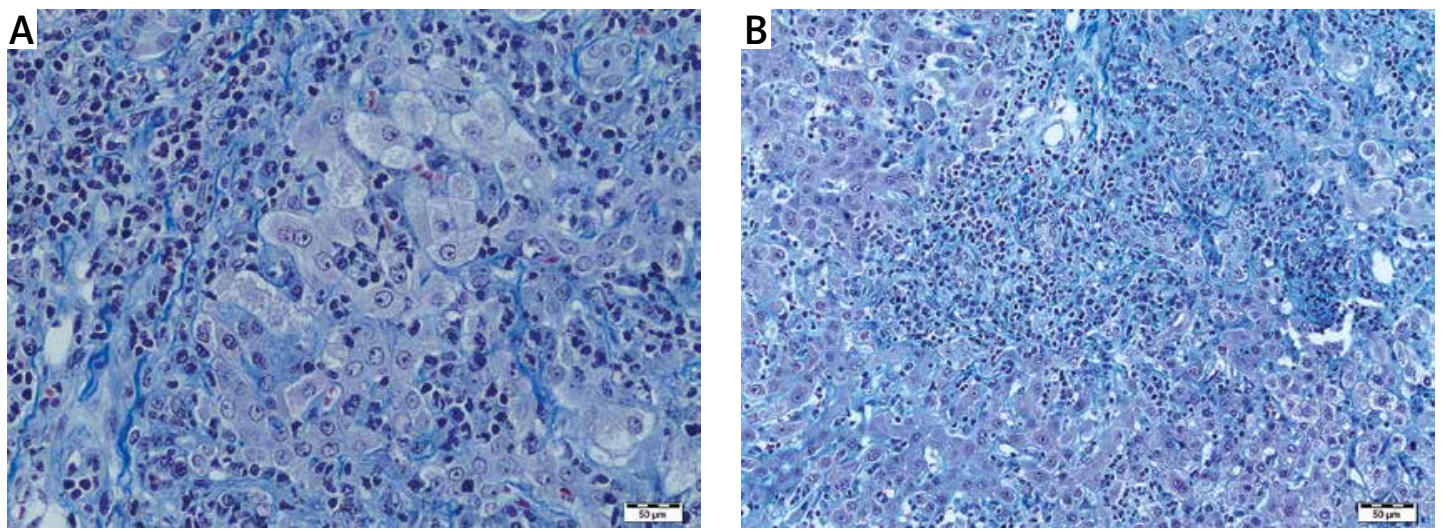

Figure 3. Patient 22 (female) (A and B): autoimmune hepatitis, inflammatory infiltrates with plasma cells, interface hepatitis

syndrome as a variant of major immune hepatopathies [3, 14].

In the cases of fatty liver disease, liver biopsy enabled assessment of the grade and stage of the inflammation, fibrosis, hepatocyte damage, and also the presence of Mallory bodies, showing a possibility of $\mathrm{ASH}$, even if alcohol consumption was not mentioned. The microscopic analysis of biopsy specimen made it possible to establish steatosis zonality, fat droplet size (macro and microvesicular), inflammation, cellular injury and fibrosis. In the present trend of non-invasive assessment of liver fibrosis, liver biopsy is still required in at least $7.2 \%$ of patients with chronic liver diseases [1]. A major limitation of liver biopsy is lack of representation as a whole organ, depending on sampling variability [1]. Usually it collects a fragment of $1 / 50000$ of the overall size of the liver (Scott). In the remaining 8 cases the microscopic changes were unspecific and did not clear the abnormal state of the liver [15]. Vanishing bile duct syndrome as ductopenia without any signs of inflammation was found in one patient with suspicion of primary biliary cirrhosis, in the absence of autoantibodies. It is also known that $5 \%$ of patients with PBC are negative for serum anti mitochondrial autoantibodies [3]. Vanishing bile duct syndrome associated with cholestasis may also include a variety of autoimmune disorders associated with progressive destruction of bile ducts [16]. The detection of iron overload in the liver biopsy was an important sign and risk factor in liver disease progression. Such excessive accumulation of body iron, especially in liver cirrhosis, is an important predictor of liver failure [17]. In one liver biopsy specimen we found normal liver architecture without inflammation or steatosis in a patient with elevated ALT and GGT, negative for viral antibodies and autoantibodies. These negative findings show that liver pathology by routine microscopic examination is not sufficient and requires further research.
In our group liver biopsy was the tool making it possible to establish the diagnosis of the disease and recommend proper treatment. In patients with steatohepatitis liver biopsy that showed fibrosis and inflammation of the liver was a strong argument for changing the life style. In 7 cases liver biopsy demonstrated autoimmune disease, in 5 cases without autoantibodies detectable in the blood. The clinical presentation of $\mathrm{AlH}$ is wide, ranging from absent or mild symptoms to fulminant hepatitis [18]. One patient only after finding Mallory bodies in his liver admitted a drinking problem and started therapy. Even in cases where the result was inconclusive biopsy made it possible to exclude some conditions, such as autoimmune hepatitis or fatty liver disease. In patients with known conditions, such as hepatitis C, not included in this paper, we have some cases of different diseases, such as hemochromatosis detected in liver biopsy during evaluation before interferon treatment.

In conclusion, despite the increasing access to new, non-invasive methods of assessment of liver fibrosis, liver biopsy remains a useful method in the differential diagnosis of liver diseases. A major limitation of liver biopsy is lack of representation as a whole organ depending on sampling variability.

\section{Conflict of interest}

The authors declare no conflict of interest.

\section{References}

1. Amarapurkar D, Amarapurkar A. Indications of liver biopsy in the era of noninvasive assessment of liver fibrosis. J Clin Exp Hepatol 2015; 5: 314-9.

2. Castera L, Vergniol J, Foucher J, et al. Prospective comparison of transient elastography, Fibrotest, APRI, and liver biopsy for the assessment of fibrosis in chronic hepatitis C. Gastroenterology 2005; 128: 343-50.

3. Dezsofi A, Baumann U, Dhawan A, et al. Liver biopsy in children: position paper of the ESPGHAN Hepatology Committee. J Pediatr Gastroenterol Nutr 2015; 60: 408-20. 
4. Filingeri V, Francioso S, Sforza D, Santopaolo F, Oddi FM, Tisone G. A retrospective analysis of 1.011 percutaneous liver biopsies performed in patients with liver transplantation or liver disease: ultrasonography can reduce complications? Eur Rev Med Pharmacol Sci 2016; 20: 3609-17.

5. Gebo KA, Herlong HF, Torbenson MS, et al. Role of liver biopsy in management of chronic hepatitis C: a systematic review. Hepatology 2002; 36(5 Suppl 1): S161-72.

6. Liberal R, Grant CR. Cirrhosis and autoimmune liver disease: current understanding. World J Hepatol 2016; 8: 1157-68.

7. Mustafa G, Alam S, Al Mamun A, Ahmad N, Alam K, Khan M. Percutaneous liver biopsy: technique and safety. Hepatogastroenterology 2011; 58: 529-31.

8. Piscaglia F, Salvatore V, Mulazzani L, Cantisani V, Schiavone C. Ultrasound shear wave elastography for liver disease. a critical appraisal of the many actors on the stage. Ultraschall Med 2016; 37: 1-5.

9. Pokorska-Spiewak M, Kowalik-Mikolajewska B, Aniszewska M, Pluta M, Marczynska M. Is liver biopsy still needed in children with chronic viral hepatitis? World J Gastroenterol 2015; 21: 12141-9.

10. Putra J, Toor A, Suriawinata AA. The utility of repeat liver biopsy in autoimmune hepatitis: a series of 20 consecutive cases. Pathology 2016; 48: 449-53.

11. Reau NS, Jensen DM. Vanishing bile duct syndrome. Clin Liver Dis 2008; 12: 203-17.

12. Reeder SB, Sirlin CB. Quantification of liver fat with magnetic resonance imaging. Magn Reson Imaging Clin N Am 2010; 18: 337-57.

13. Rust C, Beuers U. Overlap syndromes among autoimmune liver diseases. World J Gastroenterol 2008; 14: 3368-73.

14. Sikorska K, Bernat A, Wroblewska A. Molecular pathogenesis and clinical consequences of iron overload in liver cirrhosis. Hepatobiliary Pancreat Dis Int 2016; 15: 461-79.

15. Sporea I, Popescu A, Sirli R. Why, who and how should perform liver biopsy in chronic liver diseases. World J Gastroenterol 2008; 14: 3396-402.

16. Strauss E. Usefulness of liver biopsy in chronic hepatitis C. Ann Hepatol 2010; 9 Suppl: 39-42.

17. van Gerven NM, de Boer YS, Mulder CJ, van Nieuwkerk CM, Bouma G. Auto immune hepatitis. World J Gastroenterol 2016; 22: 4651-61.

18. Zhu X, Wang LC, Chen EQ, et al. Prospective evaluation of FibroScan for the diagnosis of hepatic fibrosis compared with liver biopsy/AST platelet ratio index and FIB-4 in patients with chronic HBV infection. Dig Dis Sci 2011; 56: 2742-9. 